\title{
RHIC pC CNI Polarimeter: Experimental Setup and Physics Results
}

\author{
I.G. Alekseev*†, A. Bravar** , G. Bunce ${ }^{\ddagger * *}$, R. Cadman ${ }^{\S}$, A. Deshpande ${ }^{\ddagger}$,

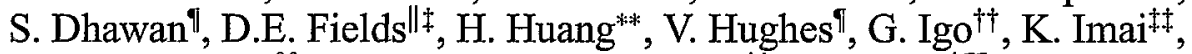

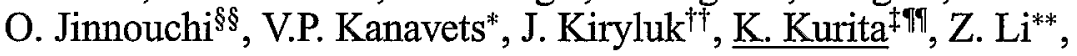 \\ W. Lozowski***, W.W. MacKay*, Y. Makdisi**, S. Rescia ${ }^{* *}$, T. Roser**, \\ N. Saito ${ }^{\ddagger \ddagger}$, H. Spinka ${ }^{\S}$, B. Surrow ${ }^{* *}$, D.N. Svirida* ${ }^{*}$ J. Tojo ${ }^{\sharp \S \S}$, \\ D. Underwood ${ }^{\S}$ and J. Wood ${ }^{\dagger \dagger}$ \\ ${ }^{*}$ Institute for Theoretical and Experimental Physics, B. Cheremushkinskaya 25, Moscow, 117259, \\ Russia \\ ${ }^{\dagger}$ E-mail: Igor:Alekseev@itep.ru \\ ** Brookhaven National Laboratory, Upton, NY 11973, USA

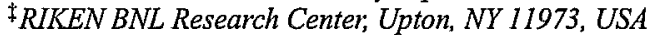 \\ $\$$ Argonne National Laboratory, Argonne, IL 60439, USA \\ "Yale University, New Haven, CT 06511, USA \\ "University of New Mexico, Albuquerque, NM 87131, USA \\ ${ }^{\dagger}$ UCLA, Los Angeles, CA 90095, USA \\ $\$$ Kyoto University, Kyoto 606-8502, Japan \\ ${ }^{\S}$ RIKEN (The Institute of Physical and Chemical Research), Wako, Saitama 351-0198, Japan \\ đI Rikkyo University, Toshima-ku, Tokyo 171-8501, Japan \\ ${ }^{* * *}$ Indiana University Cyclotron Facility, Bloomington, IN 47405, USA
}

\begin{abstract}
.
Acceleration of polarized proton beams and experiments with them at RHIC require fast and reliable measurements of the polarization.

The polarimeter presented here uses very high figure of merit of the elastic $\mathrm{pC}$ scattering at very low momenta transfer since the cross section is large. Small (a few percent) analysing power of the reaction makes it necessary to collect about $10^{7}$ events per measurement. A deadtimeless DAQ system for the polarimeter is discussed in this paper. It is based on the waveform digitizer modules with "on-board" event analysis, resulting in typical polarization measurement times of several tens of seconds.

During winter 2001/2002 RHIC polarized run several dedicated data runs were taken by the polarimeter to extract the form of the analyzing power dependence as a function of the momentum transferred at beam energies 24 and $100 \mathrm{GeV}$. This dependence is extremely important for the theoretical understanding of the CNI process including the contribution of the spin-flip hadronic amplitude. The new data may become an input to some theoretical models predicting the energy dependence of the analyzing power.
\end{abstract}

\section{INTRODUCTION}

A description of the RHIC pC CNI polarimeter is already given in this volume [1]. This paper will focus on the polarimeter DAQ in which main role is played by the wave form digitizers (WFD) and on the results of the preliminary analysis of the dedicated 
polarimeter data runs.

\section{DAQ WITH WAVE FORM DIGITIZERS}

The polarimeter operation is based on a small (about a percent) analysing power of the elastic scattering of protons on a carbon nuclei with a very small momentum transfer. Nevertheless figure of merit of this process is fairly good due to a very large crossection. This results in a requirement to collect about $10^{7}$ events in several tens of seconds. We don't like to miss events, because it will lead to unnecessary beam emittance blow up. Actually crossection is so large that we could see one good event in setup per bunch crossing. The DAQ solution to this problem was found in on-line hardware analysis of every pulse individually in each silicon strip and counting just good events.

The block diagram of the WFD module is shown in fig. 1. It contains 4 equal channels each consisting of a $140 \mathrm{MHz}$ RGB ADC, connected to Xilinx [2] FPGA, and some auxiliary logic for CAMAC interface, accelerator clock signals and FPGA power on configuration. RGB-channels of the $\mathrm{ADC}$ are used to triple digitization frequency, accepting the input signal with 3 different delays: $0,2.4$ and $4.7 \mathrm{~ns}$.

The FPGA layout which we used in the run is shown in fig. 2. Each $70 \mathrm{MHz}$ clock 6 waveform points come to the FPGA. During this run RHIC operated in 60-bunch mode and the interval between bunch crossings corresponded to exactly 15 periods of our $70 \mathrm{MHz}$ frequency, which was derived from the RHIC clock. So each waveform consists of 90 points. There were 4 possible FPGA operating modes: raw mode, subtract mode, at-mode and read-all mode. All the modes but at-mode were dedicated for debugging purposes. Here we will describe at-mode, which was used for polarimeter running. For each ADC reading pedestal subtraction was made and then the points were stored in a waveform FIFO. Simultaneously the waveform was scanned for the maximum value, which was considered as an event amplitude, and integral over the waveform was calculated. While the next waveform was moving into the FIFO the previous one was looked through for the first point above $1 / 4$ of the maximum. The number of this point gives the event time. FPGA also keeps track of the bunch number. Each bunch crossing am-

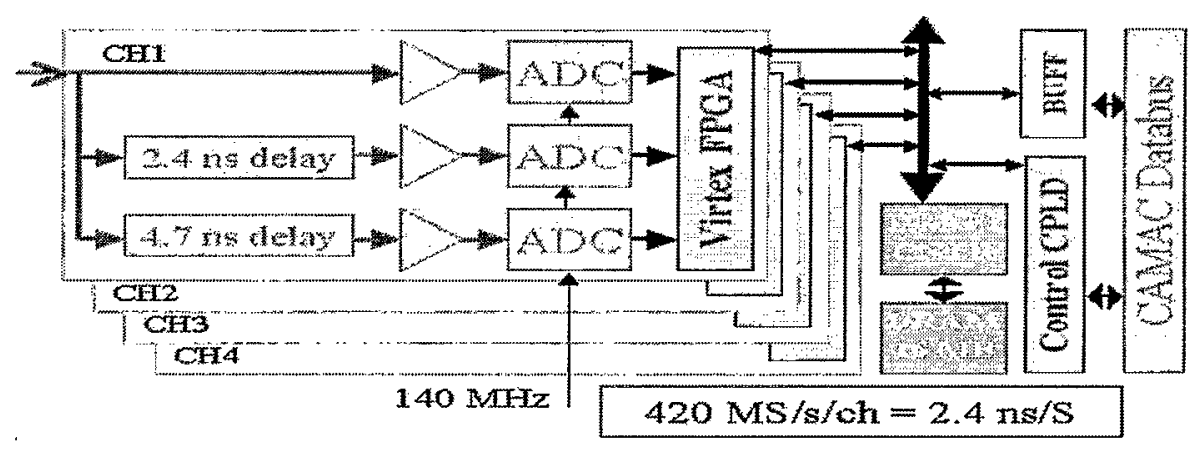

FIGURE 1. Wave form digitizer block diagram 
plitude, integral, time and bunch number formed an event. Values of time and amplitude are used as an input to the lookup table, which selected or rejected the event using time of flight to energy correlation for carbon events. Amplitude to integral lookup table was also implemented, but we didn't use it in the run. The events which passed the lookup were put into an event FIFO, which could be read through CAMAC backplane. Simultaneously these events were used to fill a set of internal histograms. This set consisted of 5 histograms: distribution of events over bunch number, energy distribution for positive bunches, energy distribution for negative bunches, energy distribution for not polarized bunches and time versus energy distribution. During polarization measurements no readout of the event FIFO was performed and only distributions were read to PC. Then the distribution of events over bunch number was used to calculate asymmetries. During the dedicated polarimeter run event data (amplitude, integral, time and bunch number) was also read to PC. The percent of events read was determined by CAMAC speed.

DAQ with WFD has no dead time and can accept events in each strip independently as often as one per bunch crossing. During the run up to $600 \mathrm{k}$ events per second were seen in the 48 active strips of the polarimeter. No data transfer was performed during the data taking in regular polarimeter runs. This results in one minute measurement time which includes $30-60 \mathrm{~s}$ of data collection and less than $10 \mathrm{~s}$ for readout and asymmetry calculation. During the RHIC polarized run polarization was measured about 1000 times.

In future we plan to adopt the FPGA design to 120-bunch mode and to make use of the onboard SDRAM memory for storage of the events.

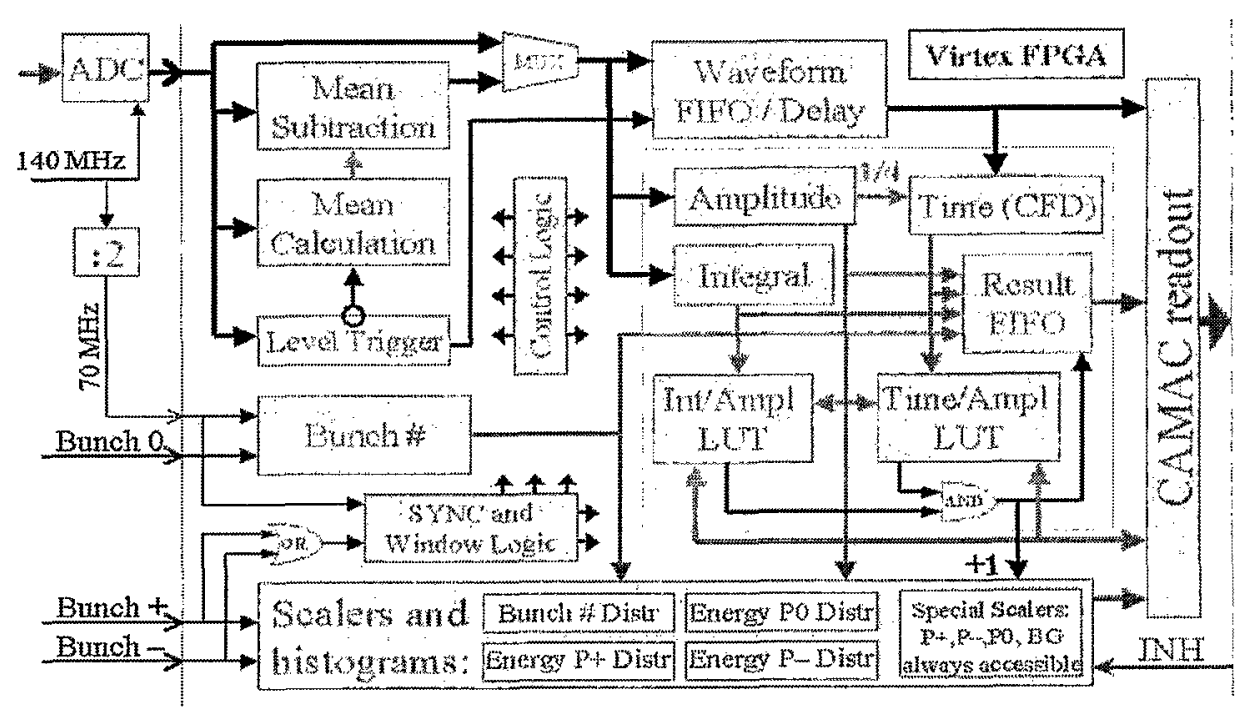

FIGURE 2. Virtex FPGA firmware block diagram 


\section{DEDICATED RUNS DATA}

During winter 2001/2002 RHIC run we took about 8 hours of running time to get several physics results. Our goals were to measure $-t$ dependence of the asymmetry, to compare it for 24 and $100 \mathrm{GeV}$ and to achieve better understanding of the carbon signal in the silicon and its processing in the readout system.

We have 8 dedicated runs at the energy 24 and $100 \mathrm{GeV}$. In 5 of them the signal was attenuated before the shaping amplifier to expand $-t$ scale. Parameters of $250 \mathrm{M}$ events were collected in addition to all distributions. These events provide us with better understanding of the selection criteria and energy distribution.

The selection of carbon events is shown in fig. 3a by outer lines in the time $(1 / \sqrt{\text { amplitude }})$ event distribution. A very careful treatment of the dead layer effect in the silicon was performed, which results in good understanding of each strip energy calibration. Some consistency check is given by extraction of the diffraction cone slope from our data. Resulting slope is shown in fig. 3b. The value $B \approx 60(\mathrm{GeV} / \mathrm{c})^{-2}$ agrees with the expectation for pC scattering $[3,4]$.

Event numbers collected inside WFD were corrected by the event distributions obtained from the at-mode data with applied cuts and then used to calculate asymmetries by the square root formula [5]:

$$
A_{p h y s}=-\frac{\sqrt{L U \cdot R D}-\sqrt{L D \cdot R U}}{\sqrt{L U \cdot R D}+\sqrt{L D \cdot R U}}
$$

where $L U, L D, R U$ and $R D$ counts in $L$ eft and $R$ ight detectors, when bunch polarization is $U \mathrm{p}$ and $D$ own correspondingly.

Asymmetry values from the new data are shown in fig. 3 for $24 \mathrm{GeV}$ (c) and $100 \mathrm{GeV}$ (d), normalized to E950 data [5]. Data from the experiment E950 at $22 \mathrm{GeV}$ is also shown in both figures. The data is fit by the formula for analysing power [6].

Here are some conclusions:

- Behavior of the asymmetry at $24 \mathrm{GeV}$ and $100 \mathrm{GeV}$ is similar but not identical.

- The data at $24 \mathrm{GeV}$ qualitatively agree with the E950 results at $22 \mathrm{GeV}$, although a statistical comparison using only the statistical errors gives a poor $\chi^{2}$. Systematic errors are being evaluated.

- The asymmetry looks to be zero at $-t>0.028(\mathrm{GeV} / \mathrm{c})^{2}$.

- Our data are not consistent with the hypothesis of the absence of the spin-flip amplitude $\left(r_{5}=0\right)$ and significantly disagree with the hypothesis of purely imaginary spin-flip $\left(\operatorname{Rer}_{5}=0\right)$.

- Resulting $\mathrm{Im} r_{5}$ and $\mathrm{Re} r_{5}$ are in a very strong correlation so can't be extracted separately from this measurement.

- Maybe energy dependent fit to our data at 24 and $100 \mathrm{GeV}$ could provide better estimation of $r_{5}$.

- It would be very interesting to expand the kinematic region to smaller $-t$, but it is difficult with current detector, because of noise and relatively thick dead layer. 


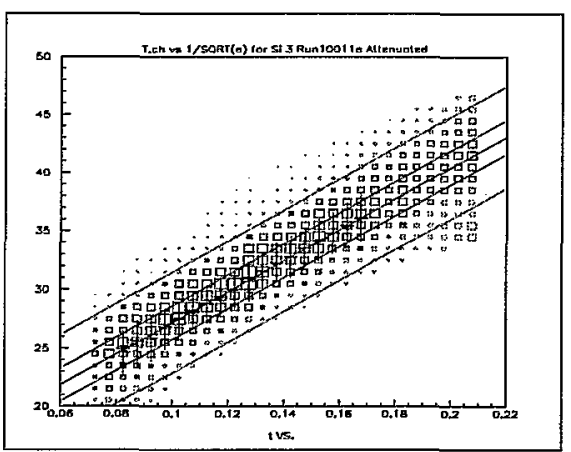

a)

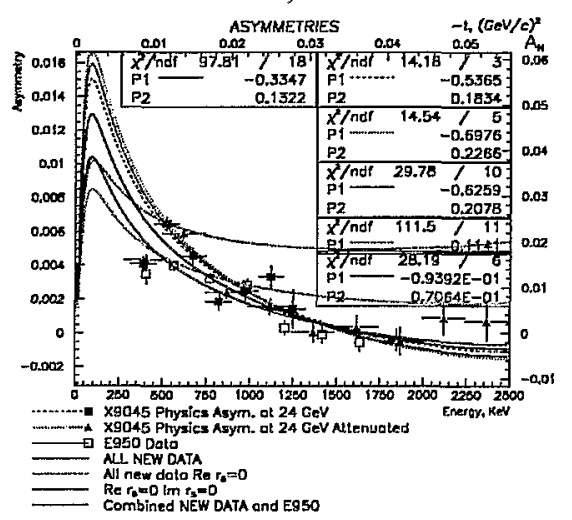

c)

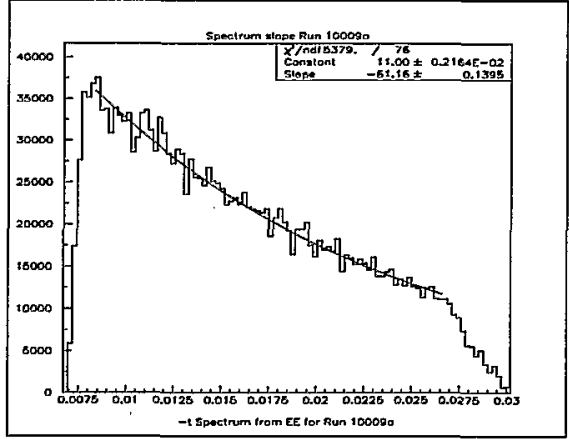

b)

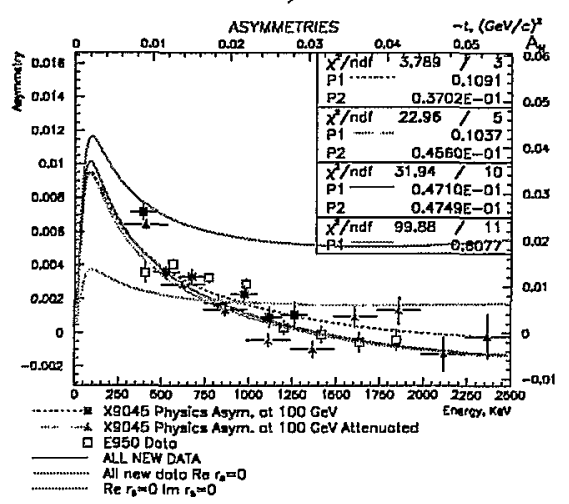

d)

FIGURE 3. a) Distribution of the events in the time versus $\frac{1}{\sqrt{\text { amplitude }}}$ plane. Outer solid lines show the cut on carbon events. b) Distribution of carbon events over 4-momentum transferred. c) Analysing power of pC elastic scattering at $24 \mathrm{GeV}$. d) At $100 \mathrm{GeV}$.

\section{ACKNOWLEDGMENTS}

We would like to thank Boris Kopeliovich and Larry Trueman for very useful discussions and help in fitting our data. We are also thankful to BNL instrumentation for preparing silicon strip detectors. US Department of Energy.

\section{REFERENCES}

1. O. Jinnouchi et al. in these proceedings.

2. http://www . xilinx.com

3. G. Bellettini et al., Nucl. Phys., P79 (1966) p. 609.

4. R.J. Glauber et al., Nucl. Phys., B21 (1970) p. 135.

5. J. Tojo et al., Phys. Rev. Lett., 89 (2002) p. 052302.

6. B.Z. Kopeliovich and T.L. Trueman, Phys. Rev., D64 (2001) p. 034004. 